\title{
Application of metabolic engineering approaches in enhancing biological hydrogen production
}

\author{
Wenfa Ng \\ Department of Chemical and Biomolecular Engineering, National University of Singapore, \\ Email: ngwenfa771@ hotmail.com
}

\begin{abstract}
Hydrogen is useful as a fuel and could be produced by a variety of means. One approach uses artificial photosynthesis where energy from sunlight powers the splitting of water into hydrogen and oxygen. But, biological methods for producing hydrogen has emerged strongly over the past decades. In particular, specific microorganisms could use different substrates to produce hydrogen at differing yields. Such fundamental discoveries with industrial applications thus motivated the use of metabolic engineering approaches and methodologies in enhancing biological hydrogen production through a series of enzyme over-expression, pathway debottlenecking, and gene deletion. However, such approaches heavily rely on the selection of an appropriate microbial chassis for biohydrogen production. With the proper strain in hand, use of alternative substrates may engender greater hydrogen productivities. But learning from the bioprocessing field, co-culture of two compatible microorganisms have been sought after for improving biohydrogen production. In addition, thermophilic microbes may also be useful candidates for exploiting hydrogen production from composting. Future outlook in the field looks into filling our gaps in understanding of the metabolic network that feeds into hydrogen production in different organisms. But, more importantly, problems such as reduced growth rate in engineered microbes point to fundamental issues with using genetically engineered microorganisms for improved biohydrogen production, to which clever bioprocess engineering may yield solutions.
\end{abstract}

Keywords: metabolic engineering, biohydrogen production, microbes, co-culture, metabolic network analysis,

Subject areas: biochemistry, microbiology, biotechnology, molecular biology, systems biology,

Formation of hydrogen removes electrons from a system; thereby, allowing coupled reaction to be driven forward. Thus, hydrogen formation reactions are useful in biotransformation for coupling with reactions that require a sink for electrons. To this end, different cellular reactions powered by different substrates enable the formation of hydrogen as electron sink. For example, dark fermentation of sugars such as glucose produces $12 \mathrm{~mol}$ of hydrogen per mol of glucose. ${ }^{1}$ Photo-fermentation of organic acids such as acetic acid, on the other hand, delivers $4 \mathrm{~mol}$ of hydrogen per mol of acetic acid. However, practical yield of hydrogen in biological hydrogen production processes is often lower; for example, 4 mol of hydrogen could be produced per mole of glucose due to the synthesis of byproducts such as acetate and butyrate. ${ }^{1,2}$ This could be due to product inhibition as the metabolism of the hydrogen producer is inhibited at high hydrogen concentration. ${ }^{3}$ It is interesting to note that 
dark fermentative production of hydrogen is more efficient and faster than hydrogen production through photosynthetic means, and the process is stable over several tens of days. ${ }^{1}$ Another natural microbial process that produces hydrogen is nitrogen fixation, where nitrogenase generates ammonia with hydrogen as by-product. ${ }^{4}$

More broadly, hydrogen could be used as a substrate for regenerating NADPH. ${ }^{5}$ Serving as a cheap reducing agent, hydrogen transfer a hydride to NADPH while a proton was transferred to solution to be taken up by a substrate. ${ }^{5}$ Key advantages of the system is $100 \%$ atom efficiency with no generation of side products. ${ }^{5}$ The approach was first demonstrated in 1981 with the regeneration of NADPH with electrons from hydrogen using a hydrogenase containing extract from Methanothermobacter thermoautotrophicus. ${ }^{5}$ However, many hydrogenase are not tolerant to oxygen and this limits process application. To this end, special focus has been dedicated to the oxygen tolerant $\mathrm{NAD}^{+}$-reducing [NiFe] hydrogenase from Ralstonia eutropha, which is highly active in the presence of oxygen at ambient oxygen concentration. ${ }^{5}$ More important, hydrogen oxidation activity only decreased by $20 \%$ at $60 \%$ oxygen $(690 \mu \mathrm{M}) .5$ Immobilized cells of $R$. eutropha has also been used and retained $60 \%$ of the initial activity after 1 week of incubation at $25^{\circ} \mathrm{C} .{ }^{5}$

Hydrogenase is naturally present in many species of anaerobic bacteria and archaea. ${ }^{6}$ Amongst microorganisms, members of the Enterobacteriaceae family are noted for their excellent fermentative hydrogen production capabilities. ${ }^{7}$ Other hydrogen producers include sulphate-reducing bacteria, homoacetogens, and methanogens. ${ }^{3}$ For example, in the presence of methanogen, syntrophic homoacetogens oxidizes sugars and lactate to acetate and hydrogen, while on their own, they reduce $\mathrm{CO}_{2}$ with hydrogen to form acetate. ${ }^{3}$ Another example would be the production of hydrogen by Desulfovibrio during growth on lactate and sulphate. ${ }^{6}$ Finally, shift of aerobic oxidation to anaerobic fermentation such as hydrogen or formate production has been known to reduce the $\Delta \mathrm{G}$ available to cells; thus anaerobic strategies could be enhanced by stripping off inhibitory product such as hydrogen. ${ }^{8}$ Hydrogen is poorly soluble in water with maximum solubility at atmospheric pressure and room temperature of $1 \mathrm{mM}^{6}$

\section{Use of alternative substrate for biological hydrogen production}

Besides glucose, other sugars and carbon sources have been explored as substrates for biological hydrogen production such as in dark or mixed acids fermentation. ${ }^{9}$ For example, glycerol could be fermented by Escherichia coli into hydrogen with a theoretical yield of $7 \mathrm{~mol}$ of hydrogen per mole of glycerol. ${ }^{1}$ An initial concentration of glycerol of $10 \mathrm{~g} / \mathrm{L}$ has been found to be optimal for $E$. coli cell growth and hydrogen production. ${ }^{1}$ Higher concentrations of glycerol have been found to depress hydrogen production. ${ }^{1}$ Both crude and pure glycerol have been found to improve hydrogen production. ${ }^{1}$ Specifically, in crude glycerol fermentation, hydrogen, acetate and butyrate were the products. ${ }^{1}$ Co-fermentation of different carbon source have also been found to increase hydrogen production. For example, co-fermentation of glycerol and glucose increased hydrogen yield by 2 -fold. ${ }^{1}$ On the other hand, addition of formate into medium with glycerol also increased hydrogen production. ${ }^{1}$ Acetate could be 
converted by anaerobic microbes to hydrogen and carbon dioxide, but only under low partial pressure of hydrogen maintained by methanogens. ${ }^{6}$

\section{Metabolic engineering approaches for enhancing biohydrogen production}

While process optimization has been useful for improving hydrogen yield, metabolic engineering approaches could theoretically enhance biological hydrogen production further. For example, metabolic pathways in E. coli were redirected to induce the DHAP production pathway and to block lactic acid, acetic acid and ethanol production. ${ }^{1}$ Such improved strains enhanced hydrogen yield to $0.68 \mathrm{mmol} \mathrm{H}_{2} \mathrm{~L}^{-1} \mathrm{~h}^{-1}$ in glycerol medium, which was 20 fold higher compared to wild-type strains. ${ }^{1}$ Pyruvate is known as a central metabolic precursor for the bioproduction of hydrogen. ${ }^{2}$ Specifically, pyruvate is converted into formate and acetyl-CoA by pyruvate formate lyase under anaerobic conditions. ${ }^{2,3}$ Lactic acid is also produced in the process. ${ }^{2}$ In hydrogen production, NADH is consumed through oxidation coupled through ferredoxin to hydrogenase. ${ }^{3}$ Formate produced is converted into hydrogen by formate hydrogen lyase $(f h l)$, resulting in a yield of 2 mol hydrogen per mol of glucose. ${ }^{2,3}$ Thus, possible targets for metabolic engineering include the deletion of lactate dehydrogenase (ldh) and fumarate reductase $(f r d B C)$ as well as the overexpression of $f h l .^{2}$ However, $f h l$ is easily inactivated under aerobic conditions even though it is the main pathway for the production of hydrogen from formate in E. coli. ${ }^{10}$ Elimination of the uptake activity of hydrogenase is also another option. ${ }^{2}$ Given that many facultative and obligate anaerobic bacteria are known to produce hydrogen, metabolic engineering approaches have also been applied in other organisms such as Enterobacter aerogenes, Enterobacter cloacae and Clostridium sp. ${ }^{2}$ For example, Clostridium $\mathrm{sp}$. have the capability of producing hydrogen as a fermentation product by removing the excess reducing powers during growth on biomass materials. ${ }^{2}$ As pyruvate is a key precursor for hydrogen production, studies have examined the pathway used by obligate anaerobic bacteria for fermenting glucose. ${ }^{2}$ Specifically, a decision-making regulatory mechanism for choosing either the Entner-Doudoroff pathway or Embden-Meyerhof pathway was postulated. In another study, a mutant strain of Enterobacter aerogenes had a 16-fold increase in hydrogen production from the NADH pathway compared to a mild increase from the formate pathway. ${ }^{2}$ Use of Citrobacter amalomaticus Y19 also resulted in the yield of $817 \mathrm{mmol} / \mathrm{L}$ of hydrogen from the pentose phosphate pathway from $100 \mathrm{mmol} / \mathrm{L}$ of glucose as substrate.

In E. coli, various genes and pathways have been manipulated for increasing biohydrogen production. To this end, overexpression of cellulases and hydrogenase were used independently or coupled with knockout of uptake hydrogenase (Hyd-1, Hyd-2 and Hyd-3) and other metabolite (e.g., succinate, lactate) producing enzymes. ${ }^{2}$ For example, E. coli strains with deleted hydrogenase (Hyd-2) showed increased hydrogen production rates, while those without Hyd-1 did not exhibit significant change in hydrogen production. ${ }^{2,10}$ More importantly, deletion of Hyd-3 abrogated hydrogen production, while deletion of Hyd-1 and Hyd-2 improved hydrogen production. ${ }^{2,11}$ As formate is the direct precursor to hydrogen production, efforts have also been expended in engineering the metabolic flux of formate. Specifically, $f h 1 A$ overexpression and deletion of $h y c A$ lead to a 2.8 fold increase in hydrogen production compared to wild-type strains. ${ }^{2}$ In addition, formate dehydrogenase that consumes formate have also been deleted, but hydrogen production decreased..$^{2}$ In another approach, heterologous 
expression of bidirectional hydrogenase from cyanobacterium increased hydrogen yield by about 41 fold compared to wild-type strains. ${ }^{2}$ On the other hand, expression of transporters for enzymes did not result in enhanced hydrogen production. ${ }^{2}$. From a metabolite perspective, disruption of succinate and lactate-producing pathways resulted in an increased yield of hydrogen gas. ${ }^{2}$ Reducing the flux of pyruvate consuming reactions is also another method used in enhancing hydrogen production. For example, deletion of pyruvate dehydrogenase (aceE) increased hydrogen yield but deletion of pyruvate oxidase (poxB) did not. ${ }^{2}$

Synthetic pathways have also been constructed for increasing biohydrogen production. For example, overexpression of proteins YdbK from E. coli, [4Fe-4S]-ferredoxin from $C$. pasteurianum, and $\mathrm{HydA}$, HydE, $\mathrm{HydF}$, and $\mathrm{HydG}$ from C. acetobutylicum increased hydrogen yield by 9 -fold. ${ }^{2}$ Transcriptional activators have also been targeted for increasing hydrogen production. For example, inactivation of NarL (the global transcriptional repressor for structural genes such as FHL, PFL and nickel transporter mik operon) increased molar yield of hydrogen by 2 -fold. ${ }^{2}$ Overexpression of $f n r$, another global transcriptional regulator, and knockout of $f o c A$ resulted in a 5.5 fold increase in hydrogen yield. ${ }^{2}$

\section{Alternative microbial chassis for biohydrogen production}

Besides E. coli, Clostridium sp. have also been engineered for increasing hydrogen production. For example, hydrogen gas was not produced when hydA gene was disrupted in $C$. perfringens. ${ }^{2}$ Furthermore, deletion of acetate kinase gene (ack) from C. tyrobutyricum increased hydrogen yield by $50 \%$. Another organism used in biohydrogen production comes from Enterobacter sp. ${ }^{2}$ Deletion of hycA and hybO encoding FHL repressor and uptake hydrogenase resulted in higher hydrogen yield, but cell growth rate decreased. ${ }^{2}$ On the other hand, heterologous expression of hydA in E. aerogenes led to a significant enhancement in hydrogen yield. Similarly, formate supplementation led to higher hydrogen production in $E$. aerogenes. ${ }^{2}$ Overexpression of $f d h F$ and $f h l A$ resulted in increased hydrogen production. ${ }^{2}$ Deletion of $l d h A$ also resulted in higher hydrogen yield. ${ }^{2}$ Overall, reduction in acids and alcohol production in Enterobacter sp. increased hydrogen yield by 1.5 fold. $^{2}$

\section{Use of co-culture technique in biohydrogen production}

In addition to metabolic engineering approaches for increasing biohydrogen production, use of co-culture approach or combination of dark fermentation and photofermentation has also been explored. ${ }^{2}$ There are two main approaches for co-culture used in biohydrogen production, (i) use of one microbial species to produce an intermediate substrate for another species to produce hydrogen, and (ii) co-culturing of dark fermentation and photofermentation bacteria. ${ }^{2}$ For example, E. aerogenes has been co-cultured with Clostridium sp. and a photofermentation bacteria for increasing hydrogen production. ${ }^{2}$ In addition, Saccharomyces cerevisiae was used in producing formate, which was subsequently used by $E$. coli for hydrogen production. ${ }^{2}$ In another example, 2 -fold increase in hydrogen yield from sucrose was observed when the fermented liquor was subjected to photofermentation. ${ }^{2}$ 
Hydrogen is also produced as a by-product such as in cyanobacteria during $\mathrm{N}_{2}$ fixation or due to dark fermentation of storage products accumulated during daytime photosynthesis. ${ }^{12}$

\section{Thermophiles as microbial candidates for producing hydrogen}

Besides co-culture approaches, thermophilic bacteria have also been explored for hydrogen production. Specifically, high hydrogen production at elevated temperatures, high molar yield, and less array of end products are useful features endowed in thermophilic bacteria. For example, knockout of ldh in Thermoanaerobacterium aotearoense prevented the accumulation of lactate and increased hydrogen yield by 2 -fold. ${ }^{2}$ In another thermophilic bacterium, yield of 4 mol hydrogen per mole of glucose was achieved in Thermotoga maritima MSB8 and DSM3109. ${ }^{2}$

\section{Future outlook}

Current gaps in understanding in the field include the relative lack of knowledge of the metabolic network of hydrogen producing reactions. ${ }^{2}$ Such understanding would help us identify the nodes for pathway engineering that could result in higher hydrogen yield without interfering with organism growth. ${ }^{2}$ In addition, various gene knockouts were found to behave differently in different microbial species. ${ }^{2}$ More importantly, genetic engineering approaches have been associated with reduced cell density, slowness in growth rates of organism, metabolic imbalance, incompatibility of heterologous pathway with native ones, and accumulation of undesired metabolites. ${ }^{2}$ Overall, besides improvement in yields, current production level of hydrogen by bacterial species remain economically unfeasible. ${ }^{2}$

\section{Conflicts of interest}

The author declares no conflicts of interest.

\section{Funding}

No funding was used in this work.

\section{References}

1 Trchounian, K. \& Trchounian, A. Hydrogen production from glycerol by Escherichia coli and other bacteria: An overview and perspectives. Applied Energy 156, 174-184, doi:https://doi.org/10.1016/j.apenergy.2015.07.009 (2015).

2 Goyal, Y., Kumar, M. \& Gayen, K. Metabolic engineering for enhanced hydrogen production: a review. Canadian Journal of Microbiology 59, 59-78, doi:10.1139/cjm2012-0494 (2012).

3 Stams, A. J. M. \& Plugge, C. M. Electron transfer in syntrophic communities of anaerobic bacteria and archaea. Nature Reviews Microbiology 7, 568, doi:10.1038/nrmicro2166 (2009). 
4 Rey, F. E., Heiniger, E. K. \& Harwood, C. S. Redirection of Metabolism for Biological Hydrogen Production. Applied and Environmental Microbiology 73, 1665 (2007).

5 Lauterbach, L., Lenz, O. \& Vincent, K. A. H2-driven cofactor regeneration with NAD(P)+-reducing hydrogenases. The FEBS Journal 280, 3058-3068, doi:doi:10.1111/febs.12245 (2013).

6 Stams, A. J. M. et al. Exocellular electron transfer in anaerobic microbial communities. Environmental Microbiology 8, 371-382, doi:doi:10.1111/j.1462-2920.2006.00989.x (2006).

7 Bagi, Z. et al. Biotechnological intensification of biogas production. Applied Microbiology and Biotechnology 76, 473-482, doi:10.1007/s00253-007-1009-6 (2007).

8 Flynn, J. M., Ross, D. E., Hunt, K. A., Bond, D. R. \& Gralnick, J. A. Enabling Unbalanced Fermentations by Using Engineered Electrode-Interfaced Bacteria. mBio 1 (2010).

9 Gabrielyan, L., Sargsyan, H. \& Trchounian, A. Novel properties of photofermentative biohydrogen production by purple bacteria Rhodobacter sphaeroides: effects of protonophores and inhibitors of responsible enzymes. Microbial Cell Factories 14, 131, doi:10.1186/s12934-015-0324-3 (2015).

10 Kim, J. Y., Jo, B. H. \& Cha, H. J. Production of biohydrogen by recombinant expression of [NiFe]-hydrogenase 1 in Escherichia coli. Microbial Cell Factories 9, 54, doi:10.1186/1475-2859-9-54 (2010).

11 Redwood, M. D., Mikheenko, I. P., Sargent, F. \& Macaskie, L. E. Dissecting the roles of Escherichia coli hydrogenases in biohydrogen production. FEMS Microbiology Letters 278, 48-55, doi:10.1111/j.1574-6968.2007.00966.x (2008).

12 Nielsen, M., Revsbech, N. P. \& Kühl, M. Microsensor measurements of hydrogen gas dynamics in cyanobacterial microbial mats. Frontiers in Microbiology 6, doi:10.3389/fmicb.2015.00726 (2015).

13 Choi, O., Um, Y. \& Sang, B.-I. Butyrate production enhancement by Clostridium tyrobutyricum using electron mediators and a cathodic electron donor. Biotechnology and Bioengineering 109, 2494-2502, doi:doi:10.1002/bit.24520 (2012).

14 Moscoviz, R., Toledo-Alarcón, J., Trably, E. \& Bernet, N. Electro-Fermentation: How To Drive Fermentation Using Electrochemical Systems. Trends in Biotechnology 34, 856-865, doi:10.1016/j.tibtech.2016.04.009 (2016). 